\title{
Using the Non-Dominant Hand for Selection in 3D
}

\author{
Joan De Boeck* \\ Tom De Weyer ${ }^{\dagger}$ \\ Chris Raymaekers \\ Hasselt University, Expertise centre for Digital Media \\ and transnationale Universiteit Limburg \\ Wetenschapspark 2, B-3590 Diepenbeek, Belgium
}

Karin Coninx ${ }^{\S}$

\begin{abstract}
Although 3D virtual environments are designed to provide the user with an intuitive interface to view or manipulate highly complex data, current solutions are still not ideal. In order to make the interaction as natural as possible, metaphors are used to allow the users to apply their everyday knowledge in the generated environment. In literature, a lot of experiments can be found, describing new or improved metaphors. In our former work, we presented the 'Object In Hand' metaphor [4], which addresses some problems regarding the access of objects and menus in a 3D world. Although the metaphor turned out to be very promising, the solution shifted the problem towards a selection problem. From the insights of our previous work, we believe the non-dominant hand can play a role in solving this problem. In this paper we formally compare three well-known selection metaphors and we will check their suitability to be carried out with the non-dominant hand in order to seamlessly integrate the most suitable selection metaphor within the 'Object In Hand' metaphor.
\end{abstract}

CR Categories: H.5.2 [User Interfaces]: Input devices and strategies-Interaction styles ;

Keywords: 3D virtual Environments, Selection Metaphors, User's Experiment

\section{INTRODUCTION}

$3 \mathrm{D}$ virtual environments are applications designed to visualise and manipulate highly complex systems or data. They aim to provide a natural interface, enabling the user to examine or adjust this information. However, it appears that establishing an intuitive and easy-to-learn interaction within the world is not an easy problem to solve. In our research, we are focused on the design and evaluation of interaction paradigms, mainly used in 3D modelling environments. In our former work, as described in section 3, we developed a metaphor which provides the user intuitive access to objects or menus in a 3D environment. Although this solution turned out to be very promising, the problem of accessing objects had been partly shifted to the problem of selecting objects. As the non-dominant hand is used to 'grab' an object and create a frame of reference for the manipulation of the dominant hand, we will examine some well known selection metaphors and how the user's non-dominant hand can play a role to jointlessly integrate the selection task in the metaphor.

In the next section, we will first give a short general overview of already known solutions which inspired our research. Next, we

\footnotetext{
*e-mail: joan.deboeck@uhasselt.be

†e-mail:tom.deweyer@uhasselt.be

†e-mail:chris.raymaekers@uhasselt.be

§e-mail:karin.coninx@uhasselt.be
}

elaborate on our previous work and its shortcomings. As selection plays a major role in the scope of this work, section 4 shows the most important metaphors which are currently used in $3 \mathrm{D}$ selection. We also consider their benefits and drawbacks, both from experiments in literature as from our own experience. Next, we motivate what we aim to examine in this paper and how it can help us to improve our former findings. In the last sections, we describe our experiment and discuss the results. We end this paper by stating our conclusions.

\section{RELATED WORK}

In some applications, the techniques used to interact with the 3D environment are based upon acts we know from our everyday life, allowing to directly apply these acts in the generated environment. Other interaction techniques are inspired on 'magic', which move away from mimicking reality. Whichever of the two approaches has been chosen, the interaction mostly copies some user's previous knowledge through the use of 'metaphors'. According to Esposito et al, these metaphors can be classified in three groups [7]: navigation, object selection and object manipulation, although other classifications such as Bowman's [3] also exist. In literature, a lot of experiments can be found describing new or improved interaction metaphors, all having their strengths and weaknesses according to the particular application or setup in which they are used. In [6] the interested reader can find a comprehensive overview of the most common interaction techniques used in virtual environments. In section 4 we will give more details on current selection metaphors in $3 \mathrm{D}$.

To further improve the interaction, multimodality is seen as one of the possibilities. Since our every-day interaction with the real world is multimodal by nature (it is a bidirectional blend of visual, aural and haptic information), it is clear that the communication with computer generated environments can be improved as well, by adopting these principles.

One of the solutions to improve the intuitiveness of the interaction is using haptic feedback. Arsenault et al [1] prove that force feedback significantly improves the performance in 3D tapping tasks. Currently, haptic feedback has proven to be useful in several application domains [16] [17]: training, tele-operations, molecular docking, virtual prototyping, etc.

Another approach to improve the naturalness of the interaction is using both hands. It is true that people use their both hands for nearly every task. Most of them are asymmetrical, which means that both hands support a different part of the global task. Guiard has created a theoretical framework for the study of this asymmetry [9]. The following principles are proposed:

- The dominant hand moves relative to the non-dominant hand. In other words, the non-dominant hand creates a frame of reference for the dominant hand. E.g., holding a sheet of paper while writing.

- The non-dominant hand's movements are low in spatial and temporal frequency, while the movements of the dominant hand are more precise and faster. 


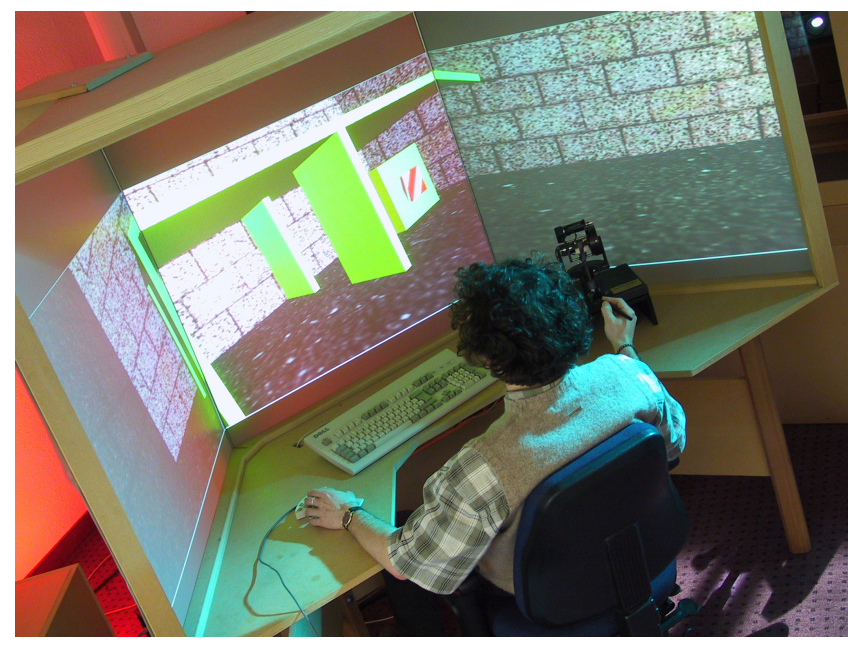

Figure 1: Personal Surround Display.

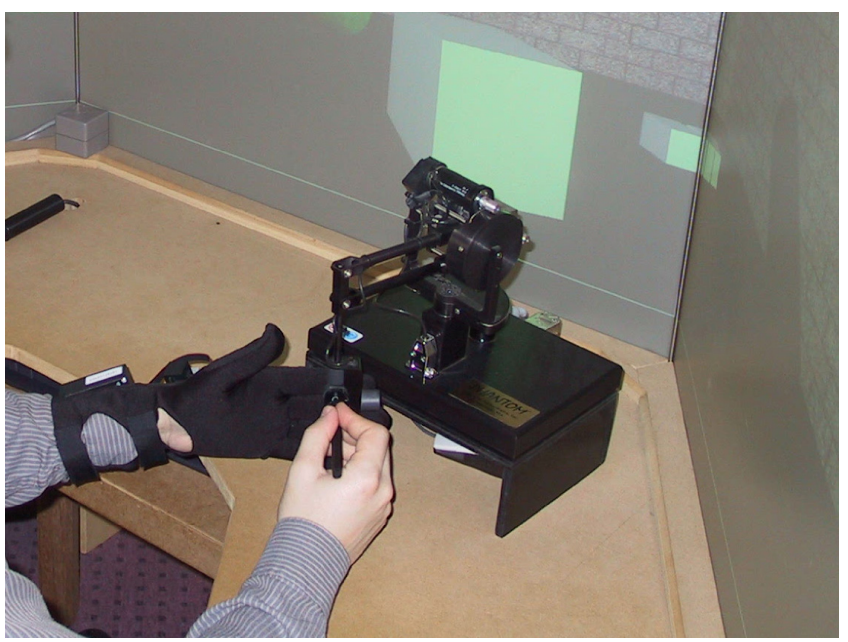

Figure 2: Non-dominant brought close to the dominant hand.
- The action of the non-dominant hand in the global bimanual task starts earlier than the dominant hand's movement. This is obvious since the non-dominant hand first has to create a reference frame before the other hand can start its task.

In the 'Voodoo Dolls' interaction technique, Pierce et al [13] exploits these principles by applying the user's both hands in order to manipulate a doll (object) in the dominant hand relative to another doll held in the non-dominant hand.

Bimanual interaction and Guiard's Kinematic Chain Model brings us to the notion of proprioception: humans have a strong kinesthetic sense of where their limbs are relative to their body. Hinckley et al prove that this sense is even independent of visual feedback [10]. The user's proprioceptive knowledge can be exploited in order to improve the interaction with the 3D world. By attaching hand-held widgets and menus to a position relative to the human's body, it is easy to rapidly find those tools [12].

\section{Previous Work}

In our earlier experiments, we found that accessing objects or menus floating in 3D space is a difficult action. Although stereo vision can address this problem [2], the use of stereo projection is not always an option. In our lab, we have a relatively low-cost semi-immersive desktop setup, which we call 'Personal Surround Display' (PSD). This setup consists of three non-stereo projection screens as shown in figure 1 .

In order to address the difficulties that users encounter when accessing objects, we adopted force feedback together with the aforementioned principles of two-handed interaction and proprioception. This led to a bimanual interaction metaphor, called 'object in hand'. The metaphor allows a user to easily activate a menu [5] or extract a selected object from its local context and bringing it in a central position [4]. The metaphor is activated by moving the non-dominant hand close to the dominant hand (figure 2). When a stretched hand is brought close, a menu is activated, and the user can interact with it, as if he is writing on a note pad. When a closed hand or fist is brought close, the selected object comes out of its context and moves to the centre of the screen, allowing the user to clutch, declutch and manipulate the object. Force feedback on the dominant hand supports a natural feeling when interacting with the object or the menu. From user experiments, we could conclude that the metaphor provides a very intuitive way to access objects. This is not a surprise, since this proprioceptive gesture is an every-day action when starting to manipulate an object.

Although the 'object in hand' metaphor has been evaluated positively, it still requires the user to select the object of interest beforehand, which more or less shifts the problem. In these former experiments, we provided the user with two selection metaphors, although the evaluation turned out that none of them was satisfying. In the next section, we will shortly describe some well-known selection metaphors in 3D environments. Next, we will describe a formal experiment we conducted in order to find the most suitable selection metaphor to integrate with the 'object in hand' metaphor. Therefore, we will also investigate the use of these metaphors with the non-dominant hand. Our conclusions will be motivated by means of this experiment.

\section{Existing Selection Metaphors}

As selection is one of the basic tasks in nearly every application, it is not surprising that a lot of work can be found about selection techniques. A comprehensive overview, with references to the original authors of the most common techniques, can be found in [3]. In this paragraph we will elaborate on three of them: virtual hand, ray or cone casting and aperture selection.

\subsection{Virtual Hand}

This interaction technique is by far the most widely known direct manipulation technique. A virtual representation of the user's hand or input device is shown in the 3D space (fig 3(a)). By moving a tracked hand or input device, the virtual representation is moved accordingly. When the virtual hand intersects an object, the object becomes selected. This metaphor has the advantage to be very simple and intuitive, since it is very similar to touching an object in real life. One of the drawbacks is that, dependent on the supported device, the the technique can be tiring with repetition. The main drawback, however, is the limited workspace in which objects can be touched. As in real life, distant objects cannot be touched. Solutions such as 'Go-Go' [15] try to solve this problem at the cost of less accuracy for distant manipulations. Alternatively, a navigation task has to precede the selection task, but dependent on the application, this is not always desirable either. 


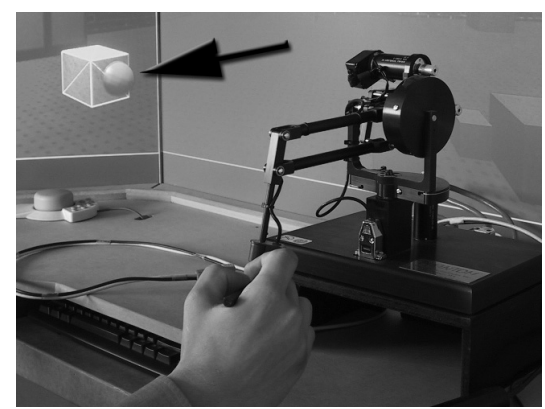

(a) Virtual Hand

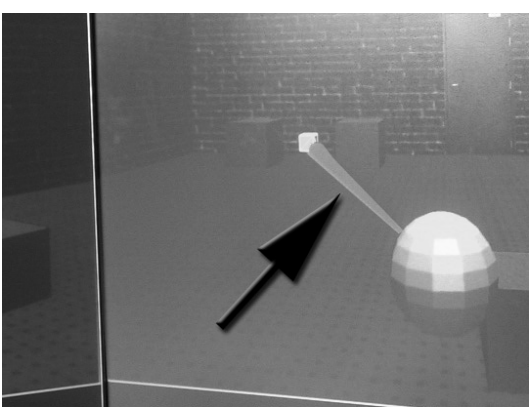

(b) Ray Casting

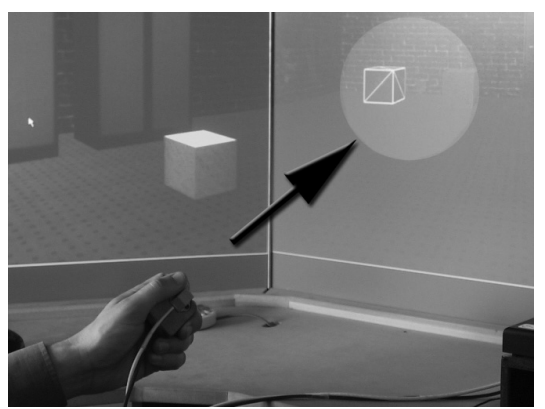

(c) Aperture Selection

Figure 3: Screenshots of three selection metaphors.

\subsection{Ray Casting or Cone Casting}

This interaction metaphor mimics the manipulation of a flash-light or laser pointer, in order to allow the user to select distant objects [11]. From the virtual pointer, a ray or a cone is casted into the world (fig 3(b)). The closest object that intersects with the ray or cone becomes selected. In this manner, distant objects that cannot be touched with a virtual hand can be accessed. However, it is clear that difficulties arise when selecting far and small objects. In several applications, ray casting turns out to be a good solution. However, if compared to a virtual hand, we could conclude from our former experiments that users rather try to avoid it [4]. A formal comparisons between virtual hand and ray selection (using the dominant hand), has been described by Poupyrev et al. [14]. Here, the ray casting shows a slightly better performance.

\subsection{Aperture Based Selection}

The aperture based selection [8] defines a cone with its apex at the user's eye point. The cone runs through the aperture, a circle floating in the world, parallel to the projection plane. By moving the aperture according to the $\mathrm{X}$ or $\mathrm{Y}$ axis, the cone is changed accordingly. By moving along the $\mathrm{Z}$ axis, the width of the cone is adjusted, as the aperture always keeps the same size. The object closest to the user, intersecting the cone, becomes selected. From the user's viewpoint, an object becomes selected when its projection falls within the aperture (fig 3(c)). In our opinion, the aperture based selection keeps the advantages of the ray selection metaphor, since it is based on the same technique of directing a cone into the world. However, the cone is not controlled by rotations, but by a translation instead, which makes it more controllable. On the other hand, still difficulties exist when accessing far and small objects.

\section{Experimental Approach}

\subsection{Motivation}

As depicted in section 3, the 'object in hand' metaphor provides the user with a very intuitive way to 'grab' an object in order to manipulate it. However, before grabbing, the object of interest must be selected first, which shifts the 'object accessing problem' to a 'selection problem'. In our former setup, we provided the user with both a virtual hand and a cone selection metaphor, but none of them turned out to be ideal because of the aforementioned reasons: the virtual hand suffered from a limited workspace and cone casting, sitting in front of a PHANToM device, turns out to be difficult for some users.

In the experiment described in this paper, we search for a better alternative in order to solve the selection problem as part of the 'object in hand' metaphor. As 'object in hand' is a two-handed interaction technique, we believe the non-dominant hand can play an important role in this solution.

Because selection is a very precise task, it is generally accepted to be performed by the user's dominant hand. However, in real life, since the non-dominant hand is used to hold an object, it is very likely that it is also used to pick the object out of its context. This brings us to the idea to involve the non-dominant hand in the selection task. As the every-day grab and hold operation is very similar to the 'object in hand' metaphor, we believe it is worth measuring the performance of the aforementioned selection techniques using the non-dominant hand.

In the next section we describe the experiment we have conducted in order to compare the selection metaphors and the performance of the dominant and the non-dominant hand. We tested the aforementioned metaphors with both hands: virtual hand, ray selection and aperture based selection. As the goal of the experiment is to use the results directly as an improvement of the 'object in hand' metaphor, we have chosen the input devices that the results immediately fit our setup. Although we are aware of the fact that this has its influence on the generality of this work.

Since force feedback improves the experience with the virtual hand selection, the PHANToM device is used for this metaphor. To compare with our former results, the PHANToM is also used for ray selection. For the aperture selection, we have chosen to use a tracker instead of the PHANToM, since this would benefit the integration, especially when we want to use it with the nondominant hand.

\subsection{Experiment}

In order to make a founded decision, we conducted a user experiment. The three aforementioned selection metaphors were tested using the dominant and the non-dominant hand. The six conditions were counterbalanced using a Latin Square design.

Twelve volunteers, ten male and two female colleagues, with little or no experience in 3D interaction, were asked to select a series of small and large objects. Some objects were positioned close by, others further away, as shown in figure 4 . The small objects were one third of the size of the large objects. The distant objects were placed at the far back side of the workspace of the virtual hand, while the objects positioned close by were positioned near the front side.

All our subjects were between the age of 22 and 31; only two of them were left handed. From a predefined list, certain objects in the scene were highlighted in an alternating way (far-close, smalllarge) in such a way that no two similar objects would be highlighted subsequently. The users were asked to select the highlighted object as efficient as possible using the offered metaphor and the demanded hand. For each condition, the subjects had to perform 16 trials from which the first 4 trials were considered as a practice. 


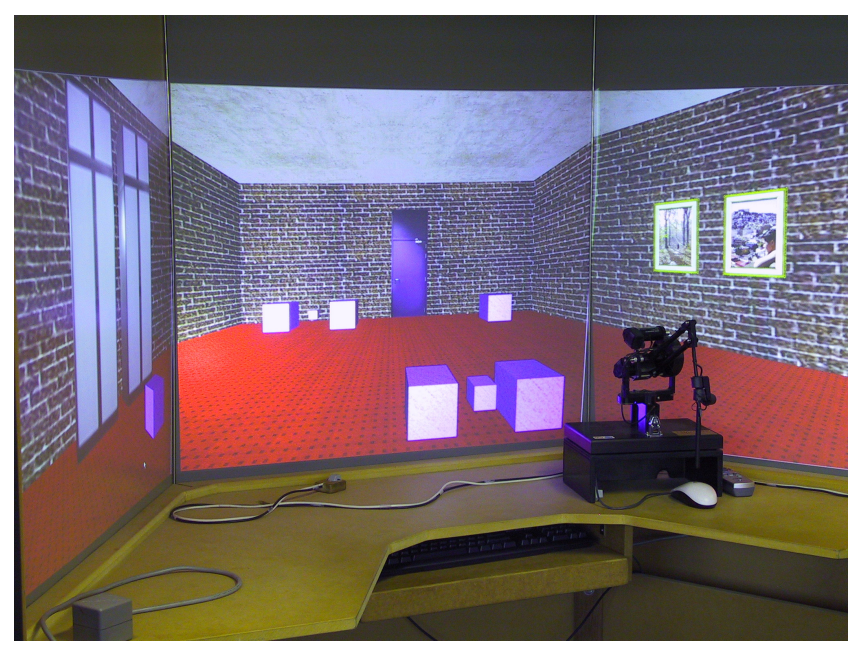

Figure 4: View of the experimental scene in the PSD

After a selection has been carried out, audio feedback is given to indicate the result (success or not). During the test, the time and result of the selection were logged. We also logged whether the object was small or large, and wether it was positioned far or close by. After the test, each volunteer was asked to give his/her subjective impressions.

\section{Results}

\subsection{General results}

Fig 5(a) shows the average result of the completion times per trial over all users for each condition. We use the following abbreviations for each condition:

- DV: Dominant Hand, Virtual Hand

- DR: Dominant Hand, Ray Selection

- DA: Dominant Hand, Aperture Selection

- NV: Non-Dominant Hand, Virtual Hand

- NR: Non-Dominant Hand, Ray Selection

- NA: Non-Dominant Hand, Aperture Selection.

To clarify the results for a first observation, a logarithmic trend line is calculated in fig 5(b). Here we see that the virtual hand metaphor is slower than the ray casting, which at its turn is slower than aperture based selection. It also appears that there is little difference between the performance of the dominant and the nondominant hand.

Table 1 compares the different conditions using one way ANOVA. While calculating the averages, the first four trials of each user were left aside, as these were meant for practicing. Considering the dominant hand, ray selection turns out to be significantly faster than virtual hand selection. Aperture based selection at its turn is significantly faster than ray selection. The same is true for the non-dominant hand. When comparing the performance of the dominant and the non-dominant hand, it is not a surprise that the non-dominant hand is slightly slower than the dominant hand, but this result is far from significant with p-values well above 0.20 . If we look at the results which are directly applicable to our setup, we see that the aperture based selection using the non-dominant hand is even significantly better than both virtual hand and ray selection

\begin{tabular}{|l|l|l|r|r|}
\hline & Time $(\mathrm{ms})$ & & Time $(\mathrm{ms})$ & P-value \\
\hline DV & 5328.68 & DR & 4207.77 & $<0.001$ \\
DR & 4207.77 & DA & 3203.95 & $<0.001$ \\
NV & 5678.80 & NR & 4228.80 & $<0.001$ \\
NR & 4228.80 & NA & 3370.39 & $<0.001$ \\
\hline DV & 5328.68 & NV & 5678.80 & 0.38024 \\
DR & 4207.77 & NR & 4228.80 & 0.94159 \\
DA & 3203.95 & NA & 3370.39 & 0.28560 \\
\hline DV & 5328.68 & NA & 3370.39 & $<0.001$ \\
DR & 4207.77 & NA & 3370.39 & 0.00140 \\
\hline
\end{tabular}

Table 1: Average completion times per condition.

with the dominant hand. The p-values are respectively $7 \mathrm{E}-13$ and 0.001 .

\subsection{Errors}

In table 2, the absolute and relative number of errors per condition are depicted. We can see that, using DR and NV, the number of errors is at its highest. The NA-condition appears to generate the lowest number of errors. With a chi-square test value of 0.41 , however, the differences are not significant. Even if we compare the best condition (NA) with the worst (DR), a chi-square value of 0.063 is not significant. Hence we can conclude that all the conditions perform equally well regarding the number of errors.

\begin{tabular}{|r|r|r|r|r|r|}
\hline & & Errors & Samples & $\%$ & Time (ms) \\
\hline $\mathrm{D}$ & $\mathrm{V}$ & 7 & 132 & $5.30 \%$ & 5328 \\
$\mathrm{D}$ & $\mathrm{R}$ & 14 & 132 & $10.61 \%$ & 4207 \\
$\mathrm{D}$ & $\mathrm{A}$ & 9 & 132 & $6.82 \%$ & 3203 \\
\hline $\mathrm{N}$ & $\mathrm{V}$ & 12 & 132 & $9.09 \%$ & 5678 \\
$\mathrm{~N}$ & $\mathrm{R}$ & 10 & 132 & $7.58 \%$ & 4228 \\
$\mathrm{~N}$ & $\mathrm{~A}$ & 6 & 132 & $4.55 \%$ & 3370 \\
\hline
\end{tabular}

Table 2: Number of errors per condition.

\subsection{Small vs Large Objects, Far vs Close Objects}

Looking at the behaviour of those metaphors in respect to the size or position of the object, other interesting conclusions can be drawn. Here, we notice no significant difference between the dominant and the non-dominant hand either. Therefore, we put the measurements of both hands together. Table 3 shows that all metaphors are significantly faster selecting large objects.

If we look at the distance of the object (table 4), surprisingly, there is no difference using the virtual hand metaphor. Ray selection, however, seems to be significantly slower when selecting objects which are close to the user. We believe this is due to the fact that the rotation of the ray plays a more important role when the objects are close by. In our former work, we already discovered that users try to avoid the rotations with this metaphor. Finally, the aperture based selection appears to be faster for the close objects, although this difference is not significant.

\begin{tabular}{|l|l|l|l|}
\hline & Small & Large & P-Value \\
\hline Virtual Hand & 5973.09 & 4970.91 & 0.012 \\
Ray Selection & 4465.84 & 3767.72 & 0.011 \\
Aperture Selection & 3791.92 & 2785.42 & $1 \mathrm{E}-11$ \\
\hline
\end{tabular}

Table 3: Comparison between small and large objects 


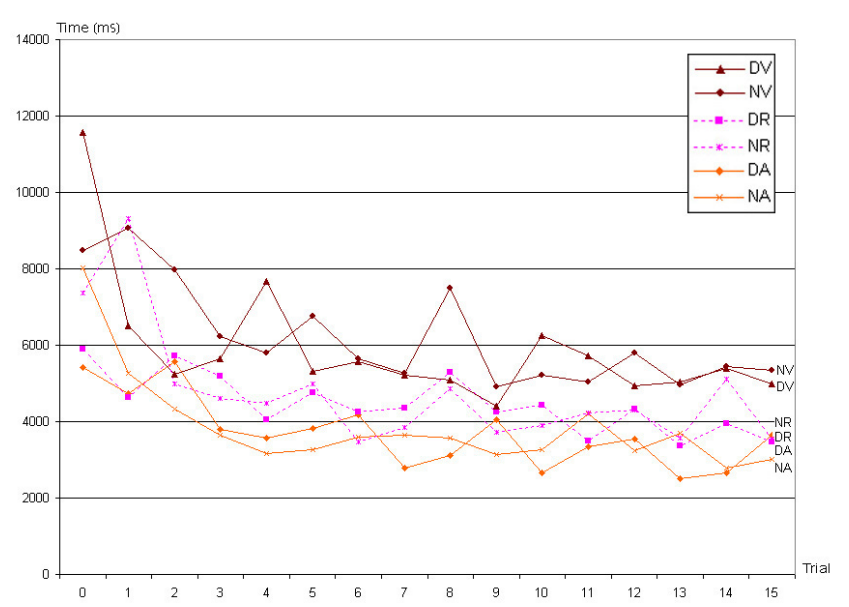

(a) Raw data.

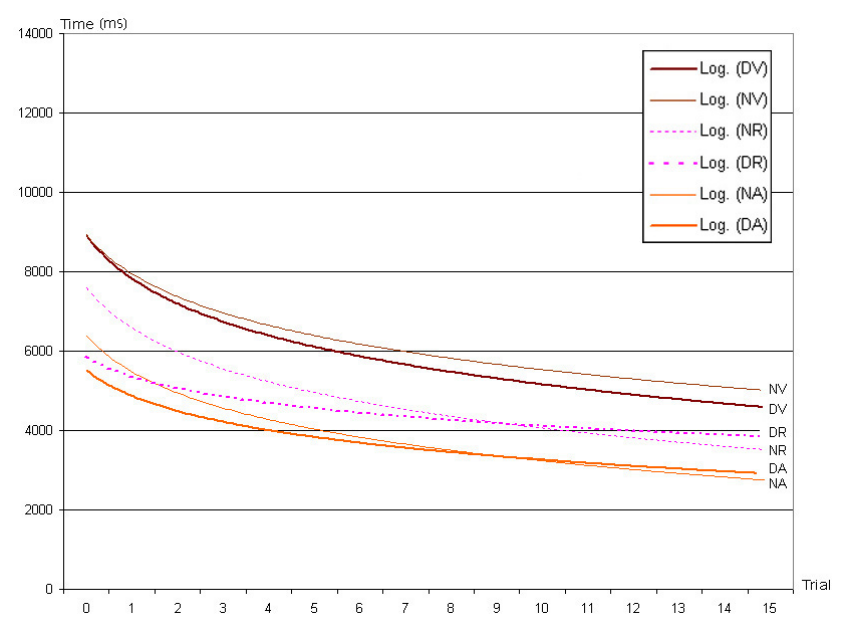

(b) Logarithmic trend line.

Figure 5: Average results for all subjects per trial.

\begin{tabular}{|l|l|l|l|}
\hline & Far & Close By & P-value \\
\hline Virtual Hand & 5532.97 & 5495.43 & 0.925 \\
Ray Selection & 3791.88 & 4645.68 & 0.003 \\
Aperture Selection & 3410.02 & 3164.33 & 0.114 \\
\hline
\end{tabular}

Table 4: Comparison between far and close by objects

\subsection{Subjective Results}

After the user completed all assignments, they were asked to complete a small survey, asking for their subjective perception of the different metaphors. Subjects had to rate their 'amount of agreement' with the given statement ('It was easy for me to select objects using the following metaphor') on a scale from 0 to 10 , with 0 indicating a total disagreement. This is shown in table 5. We see a higher agreement, both in the dominant as the non-dominant hand condition, for the aperture selection. If we statistically compare DV with DA and NV and NA, we see a significance in both cases (respectively $\mathrm{p}=0.04$ and $\mathrm{p}=0.03$ ), which allows us to conclude that our subjects found it easier to select objects using aperture based selection.

\begin{tabular}{|l|r|}
\hline Condition & Score \\
\hline DV & 6.50 \\
DR & 6.33 \\
DA & $\mathbf{8 , 0 8}$ \\
\hline NV & 4.25 \\
NA & 4.08 \\
NA & $\mathbf{6 , 5 0}$ \\
\hline
\end{tabular}

Table 5: Response to the question: 'It was easy for me to select objects using the following metaphor'.

Secondly, we asked for the users' preference when they could choose one of the metaphors for their dominant and their nondominant hand. As can be seen from figure 6, for the dominant hand, five subjects preferred the virtual hand, three chose ray selection and four the aperture selection. This result is non-significant compared to the expected values (chi-square $=0.77$ ). For the nondominant hand, no one preferred ray selection, three subjects chose virtual hand, while nine preferred aperture selection. With a chisquare value of 0.005 , this choice is significant.

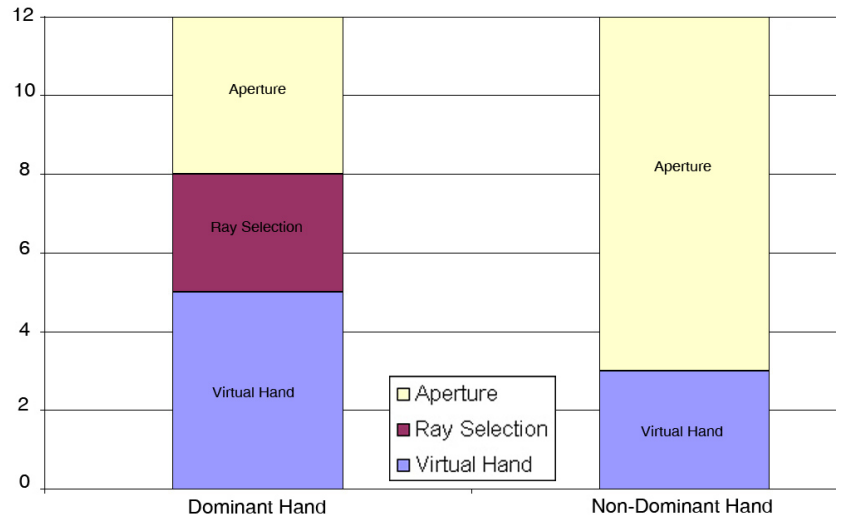

Figure 6: Subjective choice for the dominant and the non-dominant hand.

\subsection{Summary and discussion}

Aperture based selection is significantly faster than ray selection, which at its turn is significantly faster than virtual hand selection. This is both true for the dominant hand, as for the non-dominant hand. When comparing the performance of the metaphors, we find no significant difference between the two hands. Surprisingly enough, aperture selection in the non-dominant hand is even faster than the virtual hand or the ray selection using the dominant hand.

When looking into the number of erroneous selections, we cannot find a significance, allowing us to conclude that all the metaphors independent from the hand behave equally regarding the number of errors.

It turns out that all metaphors are significantly better for selecting large objects, but the results are less unambiguous when looking at the object's position. While there appears to be no significant difference between the selection of far and close objects using the virtual hand or the aperture selection, the ray selection turns out to be worse for close objects.

Finally, subjectively spoken, users claim to make selections easier using the aperture based selection. This is true for the dominant and the non-dominant hand. When users are asked to make a final choice, there is no pronounced choice for the dominant hand, 
although aperture selection is preferred for the non-dominant hand.

From these results, it turns out that in our experiment aperture selection is significantly better than the other metaphors. We believe this is true because this metaphor combines the benefits of the others, while eliminating the drawbacks. Moreover, from the user's point of view, the interaction basically appears to be $2 \mathrm{D}$, eliminating the $3 \mathrm{D}$ overhead.

\section{Conclusions}

In this paper, we presented a formal experiment which compares three well-known selection metaphors. We also searched for the difference in performance and number of errors between a selection executed with the dominant and the non-dominant hand. Although, at first glance selection seems to be an accurate task, which has to be performed by the dominant hand, we have shown some arguments why we believe a selection with the non-dominant hand can be considered.

The results of the experiment showed that the aperture based selection is in general preferable over the virtual hand or the ray selection. This also has been confirmed by a subjective questionnaire. There seems to be no significance in the number of errors between all metaphors, nor did we notice a distinction between the performance of the dominant and the non-dominant hand. Subjectively spoken, there seems to be no significant preference for the dominant hand. For the non-dominant hand, users seems to candidly prefer the aperture selection.

The results of this experiment fit into the scope of our broader research. We already presented the 'object in hand' metaphor as a solution to easily access objects and user interface elements by means of a proprioceptive gesture, although this solution shifted the problem towards a selection problem. We believe that, based upon the aforementioned results, the aperture selection using the non-dominant hand can be used in order to improve the 'object in hand' metaphor. In that case, the non-dominant hand is used to select the object and to bring the object close to the dominant hand.

\section{ACKNOWLEDGEMENTS}

Part of the research at EDM is funded by ERDF (European Regional Development Fund), the Flemish Government and the Flemish Interdisciplinary institute for Broadband technology (IBBT). The VR-DeMo project (IWT 030284) is directly funded by the IWT, a Flemish subsidy organization. This research was developed as part of our contribution in the European Network of Excellence "Enactive Interfaces" (FP6-IST 002114).

\section{REFERENCES}

[1] Roland Arsenault and Colin Ware. Eye-hand co-ordination with force feedback. In CHI 2000 conference proceedings, pages 408-414, Den Haag, NL, April 1-6 2000.

[2] Laroussi Bouguila, Masahiro Ishii, and Makoto Sato. Effect of coupling haptics and stereopsis on depth perception in virtual environments. In Proceedings of Workshop on Haptic Human-Computer Interaction, pages 54-62, Glasgow, UK, August 31 - September 12000.
[3] Doug A. Bowman, Ernst Kruijff, Joseph J. LaViola, and Ivan Poupyrev. 3D User Interfaces, Theory and Practice. Addison-Wesley, 2005.

[4] Joan De Boeck, Erwin Cuppens, Tom De Weyer, Chris Raymaekers, and Karin Coninx. Multisensory interaction metaphors with haptics and proprioception in virtual environments. In Proceedings of the third ACM Nordic Conference on Human-Computer Interaction (NordiCHI 2004), Tampere, FI, October 2004.

[5] Joan De Boeck, Chris Raymaekers, and Karin Coninx. Improving haptic interaction in a virtual environment by exploiting proprioception. In Proceedings of Virtual Reality Design and Evaluation Workshop, Nottingham, UK, January 22-23 2004.

[6] Joan De Boeck, Chris Raymaekers, and Karin Coninx. Are existing metaphors in virtual environments suitable for haptic interaction. In Proceedings of 7th International Conference on Virtual Reality (VRIC 2005), pages 261-268, Laval, France, 20-24 Apr 2005.

[7] C. Esposito. User interfaces for virtual reality systems. In Human Factors in Computing Systems, CHI96 Conference Turorial Notes, Sunday, April 141996.

[8] A. Forsberg, K. Herndon, and R. Zeleznik. Aperture based selection for immersive virtual environment. In Proceedings of UIST96, pages 95-96, 1996.

[9] Yves Guiard. Asymmetric division of labor in human skilled bimanual action: The kinematic chain as a model. In Journal of Motor Behaviour, volume 19, pages 486-517, 1997.

[10] Ken Hinkley, Randy Pausch, and Dennis Proffitt. Attention and visual feedback: The bimanual frame of reference. In Siggraph 1997: Proceedings of the 24th Annual Conference on Computer Graphics, Los Angeles, CA, USA, August 3-8 1997.

[11] Mark R. Mine. Isaac: A virtual environment tool for the interactive construction of virtual worlds. Technical Report TR95-020, UNC Chapel Hill Computer Science, ftp://ftp.cs.unc.edu/pub/technicalreports/95-020.ps.Z, may 51995.

[12] Mark R. Mine and Frederik P. Brooks. Moving objects in space: Exploiting proprioception in virtual environment interaction. In Proceed ings of the SIGGRAPH 1997 annual conference on Computer graphics, Los Angeles, CA, USA, August 3-8 1997.

[13] Jeffry Pierce, Brian Stearns, and Randy Pausch. Voodoo dolls: seamless interaction at multiple scales in virtual environments. In Proceed ings of symposium on interactive $3 D$ graphics, Atlanta, GA, USA, April 26-28 1999

[14] I. Pouprey, S. Weghorst, M. Billunghurst, and T. Ichikawa. Egocentric object manipulation in virtual environments; empirical evalutaion of interaction techniques. Computer Graphics Forum, 17(3):30-41, 1998.

[15] Ivan Poupyrev, Mark Billinghurst, Suzanne Weghorst, and Tadao Ichikawa. The go-go interaction technique: non-linear mapping for direct manipulation in vr. In Proceedings of the ACM Symposium on User Interface Software and Technology (UIST) 1996, Seattle, Washington, USA, 1996.

[16] J. Kenneth Salisbury. Making graphics physically tangible. Сотmиnications of the ACM, 42(8):74-81, August 1999.

[17] Robert J. Stone. Haptic feedback: A potted history, from telepresence to virtual reality. In Proceedings of the Workshop on Haptic Human-Computer Interaction, pages 1-8, Glasgow, UK, August 31September 12000. 